\title{
Abundances in Galactic Bulge Dwarfs and the Origin of the Elements in the Bulge
}

\section{Jennifer A. Johnson*, Andrew Gould, B. Scott Gaudi}

Department of Astronomy, Ohio State University, Columbus, Ohio, USA

E-mail: jaj, gould, gaudi@astronomy.ohio-state.edu

\section{Judith G. Cohen, Wenjin Huang}

Palomar Observatory, California Institute of Technology, Pasadena, California, USA

E-mail: jlc, wenjineastro.caltech.edu

\section{Avishay Gal-Yam}

Benoziyo Center for Astrophysics, Weizmann Institute of Science, Israel

E-mail: Avishay.Gal-yam@weizmann.ac.il

\section{Douglas C. Leonard}

Department of Astronomy, San Diego State University, San Diego, California, USA

E-mail: leonardesciences.sdsu.edu

\section{Joshua D. Simon}

Department of Astronomy, California Institute of Technology, Pasadena, California, USA

E-mail: jsimoneastro.caltech.edu

\section{Andrzej Udalski}

Warsaw Univeristy Observatory, Warsaw, Poland

E-mail: udalski@astrouw.edu.pl

\section{lan A. Bond}

Institute for Information and Mathematical Sciences, Massey University, Auckland, New Zealand

E-mail: i.a.bond@massey.ac.nz

\section{Takahiro Sumi}

Solar-Terrestrial Environment Laboratory, Nagoya University, Nagoya, Japan

E-mail: sumiestelab.nagoya-u.ac.jp 


\section{OF SCIENCE}

The Galactic Bulge has a very different chemical evolution history than the Milky Way disk or halo. The unique mix of supernovae and asymptotic giant branch stars that have contributed to the elements in the bulge offer the opportunity to identify or confirm the nucleosynthesis sites of elements. Abundance measurements based on the spectra of Galactic bulge dwarfs provide the only reliable measurements of the evolution of $\mathrm{C}$ and $\mathrm{N}$ in the Bulge, and have also been the source of the first measurements of $\mathrm{S}, \mathrm{K}, \mathrm{Zn}, \mathrm{Cu}$ and $\mathrm{Ba}$. We report on the use of gravitational microlensing to observe very faint Galactic bulge dwarfs and discuss the implications of these measurements for the origin of the elements. In particular, we argue that Type Ia SNe are unlikely to be important sources of $\mathrm{Zn}$, but while the data on $\mathrm{C}$ and $\mathrm{N}$ suggest that asymptotic giant stars contributed to the chemical enrichment of the bulge, the data on Ba suggest that there was a delay before substantial AGB star production.

10th Symposium on Nuclei in the Cosmos

July 27 - August 12008

Mackinac Island, Michigan, USA

${ }^{*}$ Speaker.

(C) Copyright owned by the author(s) under the terms of the Creative Commons Attribution-NonCommercial-ShareAlike Licence. 


\section{Introduction}

The bulge of the Milky Way has a distinct star formation history compared to the thin disk, thick disk and halo. The chemical enrichment of the bulge was dominated by Type II supernovae $(\mathrm{SNe})$ to higher metallicities than seen in the other populations. The contributions of Type Ia SNe and asymptotic giant branch (AGB) stars are not obvious until solar metallicities or higher [1], particularly when the $[\mathrm{Mg} / \mathrm{Fe}]$ ratios are considered. By comparing the abundance ratios observed in bulge stars with those in disk and halo, we can constrain the origin of elements and the timescales on which they are produced.

High-resolution spectra of main-sequence dwarf stars in the bulge are practically impossible to obtain because the faintness of the dwarfs. However, there are several elements that are observable in the spectra of dwarfs that are not in giants, either because the lines are too strong, too weak or too blended for reliable analysis (e.g., $\mathrm{Ba}, \mathrm{Zn}$, and $\mathrm{Cu}$ ) or because the surface abundances of the element have been altered by internal process and no longer reflect the original abundance (e.g $\mathrm{C}$ and $\mathrm{N}$ ). The atmospheres of dwarfs can be $\sim 500 \mathrm{~K}$ hotter than typical giant atmospheres, which makes the few lines coming from high excitation potentials stronger, the lines from low excitation potentials weaker and molecular lines much weaker. In general, the spectra are much easier to analyze. Luckily, microlensing events can magnify bulge dwarfs enough that they can be observed at the rate of about one to two a month.

\section{Microlensing Events}

When a massive body (such as another star) passes between the observer and a dwarf in the bulge, its gravity will bend the light of the dwarf. Depending on the geometry, the dwarf star can be magnified by over 100 times. This increase in brightness means that high resolution spectra can be obtained with a reasonable allocation of telescope time. However, it is critical to know which stars are being microlensed so that observations can be made within the $\sim$ half day window of high magnification. The photometric monitoring of the OGLE and MOA surveys combined with the photometric follow-up of the $\mu \mathrm{FUN}$ network make the spectroscopic observations possible.

Three highly magnified bulge dwarfs have been analyzed and published over the past two years $[2,3,4]$ and the analysis of another four are underway. These events are spread throughout the Bulge and, with one exception, are not close to the few areas in the bulge with substantial data from giants. So we are not only observing stars in different evolutionary states, but also stars in different regions (including highly reddened regions) of the bulge. We present here the results for the first three events.

\section{Abundances Ratios in Bulge Dwarfs}

In general, the abundance ratios of the $\alpha$-elements $(\mathrm{O}, \mathrm{Mg}, \mathrm{Si}$ and $\mathrm{Ca})$ in the bulge dwarfs are the same as the giants with the same $[\mathrm{Fe} / \mathrm{H}]$, although the comparison is difficult because there are few giants in the metallicity range of the dwarfs $([\mathrm{Fe} / \mathrm{H}]>0.35)$. The $[\alpha / \mathrm{Fe}]$ ratios are lower than for stars in the bulge with lower $[\mathrm{Fe} / \mathrm{H}]$, suggesting that Type Ia SNe have begun to contribute to their chemical enrichment. The $[\mathrm{S} / \mathrm{Fe}]$ ratio, measured for the first time in the bulge, is also 


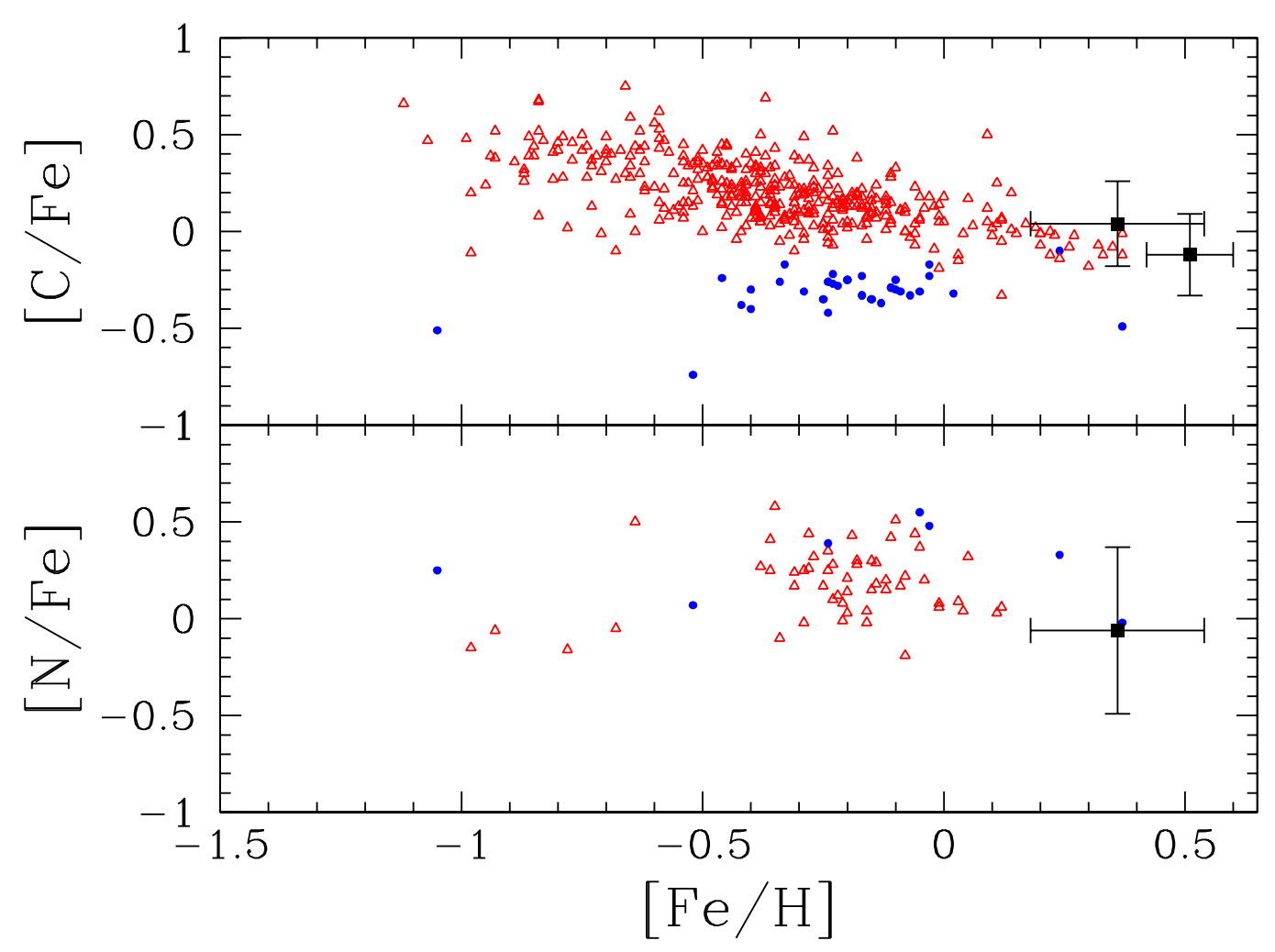

Figure 1: The abundances of $\mathrm{C}$ and $\mathrm{N}$ in the bulge. The red triangles show literature data for dwarfs in the disk and the blue circles show data for bulge giants (see [4] for a list of literature sources). The black squares are the data for the bulge dwarfs. The low $[\mathrm{C} / \mathrm{Fe}]$ values in the giants show the effects of internal mixing, which is not a factor for the dwarfs.

not enhanced, which agrees with this picture. While we have measured abundances for many elements in the bulge, we have concentrated here on five elements where the measurements in dwarfs represent the first of their kind in the bulge.

The $\mathrm{C}$ and $\mathrm{N}$ abundances in Figure 1 are the first in the bulge that reflect the chemical evolution of the bulge. The solar values of $[\mathrm{C} / \mathrm{Fe}]$ and $\mathrm{N} / \mathrm{Fe}]$ show that $\mathrm{C}$ and $\mathrm{N}$ production kept pace with the Fe production in the Bulge. There are many sources of $\mathrm{C}$ in the Universe [5, for example]. Type II SNe and AGB stars certainly contribute substantial amounts of $\mathrm{C}$ and $\mathrm{N}$; the roles of novae and Wolf-Rayet stars are less clear. These contributions, whatever they are, track the production of $\mathrm{Fe}$ in the chemical evolution of the Bulge.

Figure 2 shows the $[\mathrm{Ba} / \mathrm{Fe}]$ ratios measured in the bulge dwarfs. Stellar populations dominated by Type II SNe and r-process production should have low $[\mathrm{Ba} / \mathrm{Fe}]$ before Type Ia SNe and AGB stars eventually add $\mathrm{Fe}$ and $\mathrm{Ba}$. Whether the Ba deficiency in one dwarf can be explained by a lack of contributions from AGB stars depends on the relative timescales of Type Ia SN pollution and AGB pollution and whether the low $[\alpha / \mathrm{Fe}]$ values in the bulge dwarfs are the result of Type Ia pollution. If we assume that Type Ia SNe have contributed significantly to the abundances of the dwarfs, which is reasonable, then AGB pollution of Ba must trail Type Ia SN production. However, $\mathrm{C}$ production in AGB stars must either be completely negligible in the bulge or not subject to same 


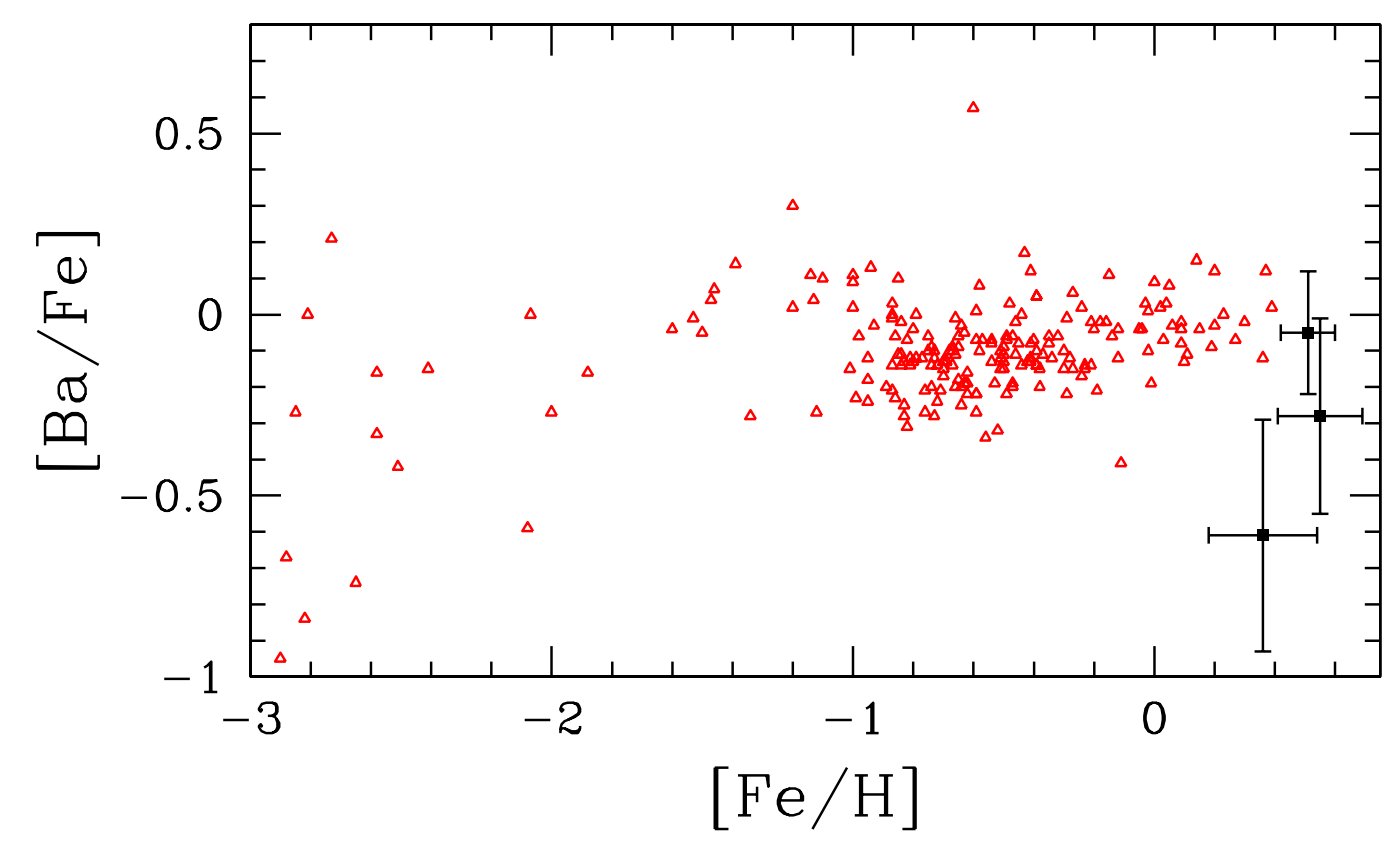

Figure 2: The abundances of $\mathrm{Ba}$ in the bulge. The red triangles are literature values for disk dwarfs; black squares are values for the bulge dwarfs. The lowest metallicity bulge dwarf has a strikingly low [Ba/Fe] ratio.

time delay, to explain the solar $[\mathrm{C} / \mathrm{Fe}]$ ratio.

Finally, Figure 3 shows the abundances of two elements just past the iron peak: $\mathrm{Cu}$ and $\mathrm{Zn}$. The source of these elements throughout the history of the Galaxy is uncertain [6, for example], but observations show that at low metallicities in the halo, these two elements have very different behaviors. The same seems to be true at the high metallicities observed in the bulge. While $[\mathrm{Cu} / \mathrm{Fe}]$ is supersolar in the high metallicity bulge dwarfs, $[\mathrm{Zn} / \mathrm{Fe}]$ is at most solar. The measurements of $[\mathrm{Cu} / \mathrm{Fe}]$ and $[\mathrm{Zn} / \mathrm{Fe}]$ (Figure 3) argue against the production of large amounts of $\mathrm{Zn}$ in Type Ia SNe, while the increase in $[\mathrm{Cu} / \mathrm{Fe}]$ could come from either metal-rich Type II SNe or Type Ia SNe.

\section{Conclusion}

High-resolution spectra from highly magnified bulge dwarfs have allowed new elements to be measured in the bulge of the Milky Way. These observations constrain the nucleosynthesis sites and production timescales of elements including $\mathrm{C}, \mathrm{N}, \mathrm{Ba}$, and $\mathrm{Zn}$. Our preliminary results suggest that Type Ia SNe do not contribute substantial amounts of Zn, and that AGB production of $\mathrm{C}$ and $\mathrm{N}$, though perhaps not $\mathrm{Ba}$, have contributed to the pollution of stars in the bulge.

The fact that the bulge dwarfs are clustered at high $[\mathrm{Fe} / \mathrm{H}]$, although the microlensing events should be independent of metallicity, is striking. The metallicity distribution function is discussed 


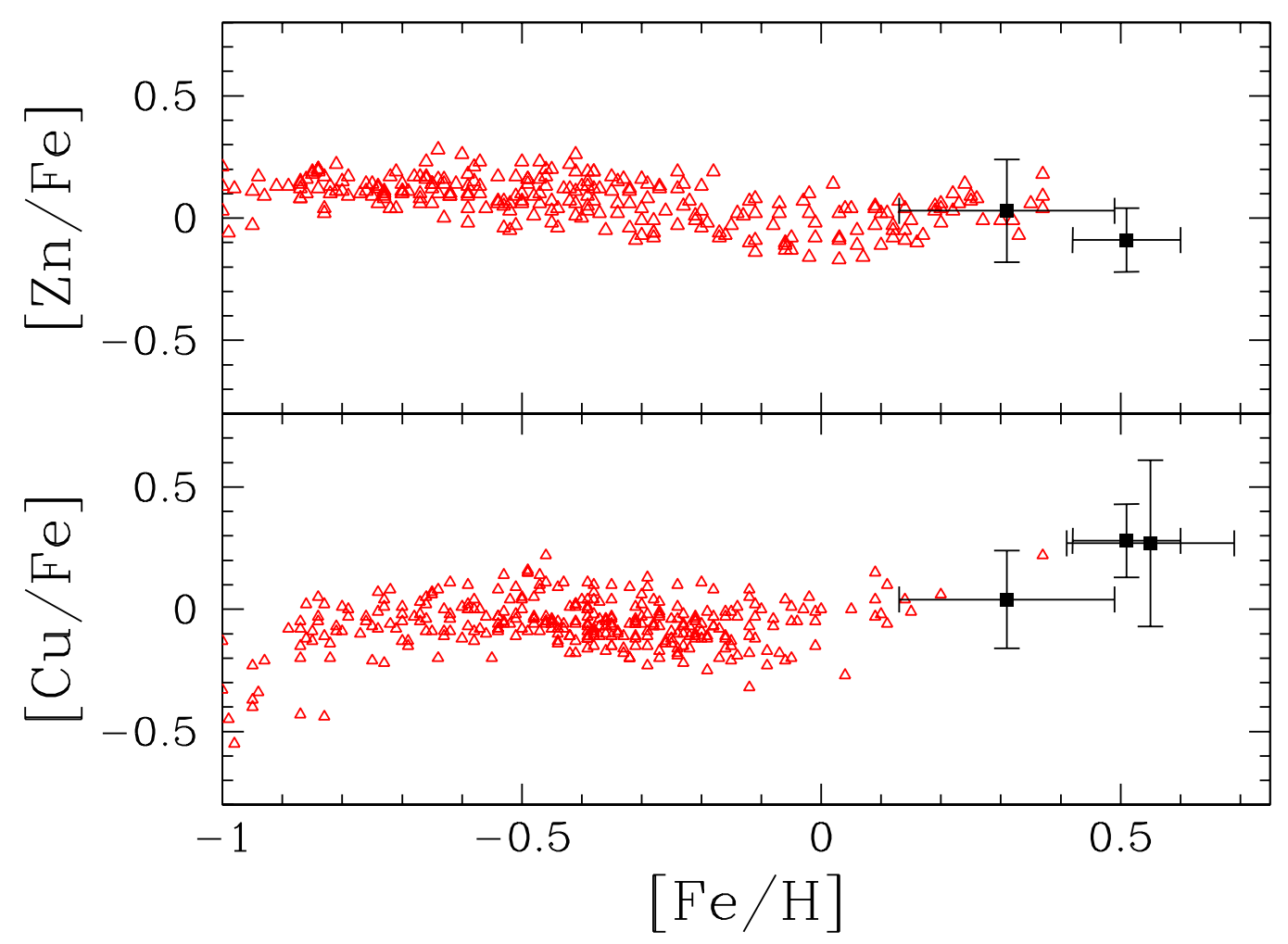

Figure 3: The abundances of $\mathrm{Cu}$ and $\mathrm{Zn}$ in the bulge. The red triangles show literature data for dwarfs in the disk and the black squares show the data for the bulge dwarfs.

in more detail in [3, 4]. Additional observations of bulge dwarfs are planned, as more data, particularly at lower metallicities, are important to trace the full evolution of these elements.

\section{References}

[1] A. McWilliam, \& R. M. Rich, 1994, The first detailed abundance analysis of Galactic bulge K giants in Baade's window, ApJS, 91, 749

[2] J. A. Johnson, A. Gal-Yam, D. C. Leonard, J. D. Simon, A. Udalski, \& A. Gould, 2007, A High-Resolution Spectrum of the Extremely Metal-Rich Bulge G Dwarf OGLE-2006-BLG-265, ApJL, 655, L33

[3] J. G. Cohen, W. Huang, A. Udalski, A. Gould, \& J. A. Johnson, 2008, Clues to the Metallicity Distribution in the Galactic Bulge: Abundances in OGLE-2007-BLG-349S, ApJ, 682, 1029

[4] J. A. Johnson, B. S. Gaudi, T. Sumi, I. A. Bond, \& A. Gould 2008, A High-Resolution Spectrum of the Highly Magnified Bulge G Dwarf MOA-2006-BLG-099S, ApJ, 685, 508

[5] B. Gustafsson, T. Karlsson, E. Olsson, B. Edvardsson, \& N. Ryde, N. 1999, The origin of carbon, investigated by spectral analysis of solar-type stars in the Galactic Disk, A\&A, 342, 426

[6] S. Bisterzo, L. Pompeia, R. Gallino, M. Pignatari, K. Cunha, A. Heger, \& V. Smith, 2005, Cu and Zn in different stellar populations: Inferring their astrophysical origin, Nuclear Physics A, 758, 284 